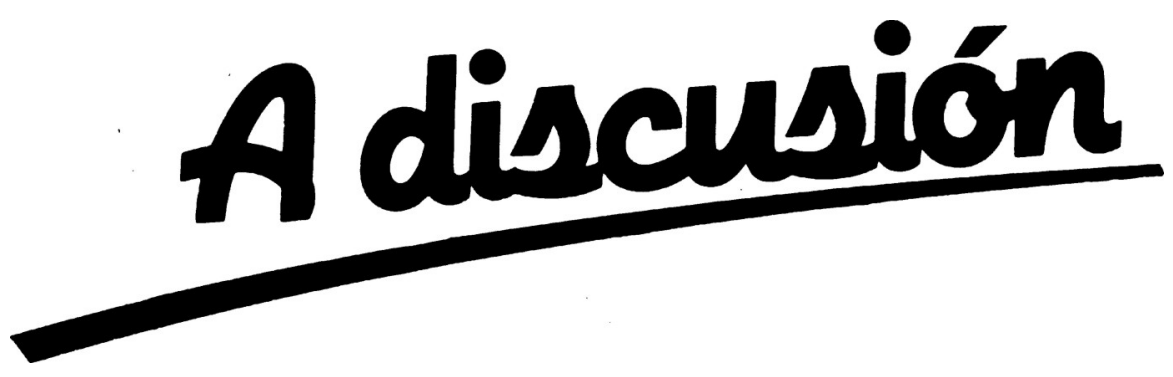

COORDINATION THROUGH DE BRUIJN SEQUENCES*

Olivier Gossner and Penélope Hernández**

WP-AD 2005-05

Corresponding author: O. Gossner. Paris-Jourdan Sciences Économiques, UMR CNRS EHESS - ENPC - ENS. 48 boulevard Jourdan. 75014 Paris. Tel.: +33 443136380 / E-mail: Olivier.Gossner@enpc.fr.

Editor: Instituto Valenciano de Investigaciones Económicas, S.A.

Primera Edición Febrero 2005

Depósito Legal: V-998-2005

IVIE working papers offer in advance the results of economic research under way in order to encourage a discussion process before sending them to scientific journals for their final publication.

* P. Hernández thanks the financial support from the Spanish Ministry of Education under project SEJ 2004-02172/ECON and the Instituto Valenciano de Investigaciones Económicas (Ivie).

** O. Gossner: Paris-Jourdan Sciences Économiques. P. Hernández: Universidad de Alicante. Dpto. Fundamentos del Análisis Económico. 


\title{
COORDINATION THROUGH DE BRUIJN SEQUENCES
}

\author{
Olivier Gossner and Penélope Hernández
}

\begin{abstract}
Let $\mu$ be a rational distribution over a finite alphabet, and $\left(\chi_{t}\right)$ be a $n$-periodic sequences which first $n$ elements are drawn i.i.d. according to $\mu$. We consider automata of bounded size that input $\chi_{t-1}$ and output $y_{t}$ at stage $t$. We prove the existence of a constant $C$ such that, whenever $m \ln m \geq C n$, with probability close to 1 there exists an automaton of size $m$ such that the empirical frequency of stages such that $y_{t}=\chi_{t}$ is close to 1 . In particular, one can take $C=\frac{\bar{p}}{1-\bar{p}} \ln \frac{1}{p}$, where $\bar{p}=\max _{\theta \in \Theta} \mu(\theta)$ and $\underline{p}=\min _{\theta \in \Theta} \mu(\theta)$.
\end{abstract}

Key words: Coordination, complexity, De Bruijn sequences, automata 


\section{Introduction}

A consequence of a Myhill-Nerode's classical theorem on the theory of regular languages (see [HMU01] for instance) is that the size of any automaton that implements a sequence of least period $n$ must be at least $n$. This result has been used to measure the complexity of strategies in repeated games played by finite automata e.g. by [AR88], [Ney97]. More generally, these games lead to study the complexity of coordination between a periodic sequence $\left(x_{t}\right)$ and an automaton that inputs $x_{t-1}$ at stage $t$.

Neyman [Ney97] proves that, if $x_{1}, \ldots, x_{n}$ are drawn i.i.d.according to any probability distribution $\mu$ over an alphabet $\Theta$, whenever $m \ln m \ll n$, with probability close to 1 there exist no automaton of size $m$ that achieves nonnegligible correlation with the sequence $x_{1}, \ldots x_{n}, x_{1}, \ldots$ This implies that in a repeated zero-sum game, there exists a sequence of size $n$ (and thus an automaton of size $n$ ) that guarantees the value of the stage game against all automata of size $m$ of the opponent if $m \ln m \ll n$.

In this article we prove that if $\mu$ is rational, there exists a constant $C$ such that, whenever $m \ln m \geq C n$, with probability close to 1 there exists an automaton of size $m$ that matches the sequence at almost every stage. In particular, one

can take $C=\frac{\bar{p}}{1-\bar{p}} \ln \frac{1}{\underline{p}}$, where $\bar{p}=\max _{\theta \in \Theta} \mu(\theta)$ and $\underline{p}=\min _{\theta \in \Theta} \mu(\theta)$. This implies that the condition $m \ln m \ll n$ in Neyman's result is (almost) tight when $\mu$ is rational.

In a previous article [GH03], we prove a similar result when $\mu$ is the counting measure. For a given sequence, the construction of an automaton in [GH03] relies on sequences for which the frequencies of all words $y_{1}, \ldots, y_{\ell}$ of length $\ell$ 
are the same (De Bruijn sequences). In the present work, we rely on generalized De Bruijn sequences, in which the empirical frequency of a word $y_{1}, \ldots, y_{\ell}$ of length $\ell$ is $\prod_{k=1}^{\ell} \mu\left(y_{k}\right)$. The assumption that $\mu$ is rational is needed for the existence of these sequences. The construction of the automaton depends on a statistical condition on the $n$ periodic sequence that we call regularity. We prove that the probability of the set of such regular sequences goes to 1 as $n$ goes to infinity using large deviations properties. This approach simplifies the computations in [GH03] that relies on counting arguments, and improves the constant $C$ when $\mu$ is uniform over a set $X\left(\frac{1}{|X|-1} \ln |X|\right.$ instead of $\left.e|X| \ln |X|\right)$.

We present the model in Section 2, and state and prove the main result in Section 3 .

\section{Model}

For $z \in \mathbb{R}$, we let $\lfloor z\rfloor$ and $\lceil z\rceil$ denote the integer part and the superior integer part of $z$ respectively $(z-1<\lfloor z\rfloor \leq z$ and $z \leq\lceil z\rceil<z+1)$. The cardinality of a finite set $Z$, is denoted $|Z|$. Let $\Theta$ be a finite alphabet, and let $\Theta_{n}$ represent the set of $n$-periodic sequences of elements of $\Theta$.

A (finite) automaton $M \in F A(m)$ of size $m$ with inputs and outputs in $\Theta$ is a tuple $M=<Q, q^{*}, f, g>$, where $Q$ s.t. $|Q|=m$ is the finite set of states, $q^{*} \in Q$ is the initial state, $f: Q \rightarrow \Theta$ is the action function, and $g: Q \times \Theta \rightarrow Q$ is the transition function.

An automaton $M \in F A(m)$ and a sequence $x=\left(x_{t}\right)_{t} \in \Theta^{\mathbb{N}}$ induce a sequence of states and actions $\left(q_{1}, y_{1}, q_{2}, y_{2}, \ldots\right)$, where $q_{1}=q^{*}, y_{1}=f\left(q^{*}\right)$,

and for $t \geq 2, q_{t}=g\left(q_{t-1}, x_{t-1}\right), y_{t}=f\left(q_{t}\right)$. The corresponding sequence of 
actions $\left(y_{t}\right)_{t \geq 1}$ chosen by the automaton is denoted $y(x, M)$. If $x^{n} \in \Theta_{n}$, then $\left(x_{t}, y_{t}\left(x^{n}, M\right)\right)_{t}$ is periodic of period at most $m n$ after a finite number of stages.

We define the ratio of coincidences between $x^{n} \in \Theta_{n}$ and $M \in F A(m)$ is:

$$
\rho\left(x^{n}, M\right)=\lim _{T \rightarrow \infty} \frac{1}{T}\left|\left\{1 \leq t \leq T: y_{t}\left(x^{n}, M\right)=x_{t}^{n}\right\}\right|
$$

$\rho\left(x^{n}, M\right)$ is the average proportion of stages for which $M$ predicts correctly the sequence $x^{n}$. Given $x^{n}$, the best ratio of coincidences that an automaton of size $m$ can achieve with $x^{n}$ is $\rho^{m}\left(x^{n}\right)=\max _{M \in F A(m)} \rho\left(x^{n}, M\right)$.

\section{$3 \quad$ Asymptotic properties}

We are concerned with asymptotic properties of the distribution of $\rho^{m}\left(x^{n}\right)$ when the first $n$ elements of $x^{n}$ are drawn i.i.d.according to some rational distribution $\mu$ in $\Delta(\Theta)$. Let $\Phi$ be a common denominator of $\left(p_{i}\right)_{i \in \Theta}$, and denote $\bar{p}=\max _{i} p_{i}, \underline{p}=\min _{i} p_{i}$. We assume wlog. $\underline{p}>0$. Pr represents the induced probability on the sets $\Theta_{n}$. Neyman [Ney97] proved the following:

Theorem 1 (Neyman 97) For a sequence $(m(n))_{n}$ of positive integers, if $\lim _{n \rightarrow \infty} \frac{m(n) \ln m(n)}{n}=0$ then:

$$
\forall \varepsilon>0, \lim _{n \rightarrow \infty} \operatorname{Pr}\left(\rho^{m}\left(x^{n}\right)<\bar{p}+\varepsilon\right)=1
$$

This result provides an asymptotic condition on $m$ and $n$, namely $\frac{m \ln m}{n} \rightarrow 0$, under which automata of size $m$ cannot achieve coordination ratios larger than $\bar{p}$ with probability close to 1 . Our main result shows the existence of a constant $C$ such that if $\frac{m \ln m}{n}$ is asymptotically larger than $C$, then automata of size $n$ can achieve coordination ratios arbitrarily close to 1 with a set of periodic 
sequences of probability close to 1 .

Theorem 2 There exists a constant $C$ such that for any sequence of positive integers $(m(n))_{m \in \mathbb{N}}$ with $\lim _{n \rightarrow \infty} \frac{m(n) \ln m(n)}{n}>C$,

$$
\forall \varepsilon, \operatorname{Pr}\left(\rho^{m}\left(x^{n}\right)>1-\varepsilon\right) \longrightarrow 1
$$

In particular, one can take $C=\frac{\bar{p}}{1-\bar{p}} \ln \frac{1}{\underline{p}}$.

To prove this, we define in Section 3.1 a subset of $\Theta_{n}$ of sequences verifying a statistical regularity condition. We call those sequences regular. Then, in Section 3.2, for each regular sequence $x^{n}$, we construct an automaton in $F A(m)$ that achieves a large ratio of coincidences with $x^{n}$. We estimate the probability of regular sequences in Section 3.3, and conclude the proof in Section 3.4.

\subsection{Regularity}

In this section we define the statistical regularity condition that ensures a large ratio of coincidences. Let $x=x^{n}=\left(x_{1}, x_{2}, \ldots\right) \in \Theta_{n}$ and $\ell \leq n$. We call word an element of $\Theta^{\ell}$. We identify $x$ to its $n$ first elements, thus making the abuse of notation $x \in \Theta^{n}$. For $1 \leq j \leq\left\lfloor\frac{n}{\ell}\right\rfloor$, we write $r_{j}=\left(x_{\ell(j-1)+1}, \ldots, x_{\ell j}\right)$ and $r^{\prime}=\left(x_{\left\lfloor\frac{n}{\ell}\right\rfloor \ell+1}, \ldots, x_{n-1}, x_{n}\right)$. This way, $x$ can be expressed as the concatenation of the words $r_{1}, \ldots, r_{\left\lfloor\frac{n}{\ell}\right\rfloor}$ and of $r^{\prime} \in \Theta^{n-\ell\left\lfloor\frac{n}{\ell}\right\rfloor}$. Let $x^{*}$ be the concatenation of $r_{1}, \ldots, r_{\left\lfloor\frac{n}{\ell}\right\rfloor}$. The number of times that a word $r$ appears in $x^{*}$ is

$$
S\left(x^{*}, r\right)=\left|\left\{0 \leq j \leq\left\lfloor\frac{n}{\ell}\right\rfloor: r_{j}=r\right\}\right|
$$

For $\alpha>1$, we define the set of $(\alpha, \ell)$-regular (or regular for short) sequences $R_{\ell}(n, \alpha)$ as the subset of elements $x$ of $\Theta_{n}$ such that for each word $r, S\left(x^{*}, r\right) \leq$ 
$\alpha \frac{n}{\ell} \operatorname{Pr}(r)$.

\subsection{Construction of an automaton for regular sequences}

Proposition 3 Let $x \in R_{\ell}(n, \alpha)$. With $m=\left\lceil\alpha \frac{\bar{p}}{1-\bar{p}} \frac{n}{\ell \Phi^{\ell}}\right\rceil \Phi^{\ell}+\ell, \rho^{m}(x) \geq 1-\frac{1}{\ell}$.

The proof of the proposition is constructive.

\subsubsection{Proof of Proposition 3}

We present the construction of an automaton $M=<Q, q^{*}, f, g>\in F A(m)$ that ensures a sufficient coincidence ratio with $x \in R_{\ell}(n, \alpha)$. First, we design $Q$ and $f$, second we define $q^{*}$ and $g$. Finally we check that $M$ achieves the desired ratio of coincidences with $x$.

\subsubsection{Construction of the state space and action function The} state space and action function we design depend only on $\mu, \alpha, n$ and $\ell$, they are independent of the particular element $x$ of $R_{\ell}(n, \alpha)$. Our construction relies on a sequence of elements of $\Theta$ such that the empirical frequencies of each word coincides with its probability under Pr. To construct this sequence, we first construct a sequence over an alphabet of size $\Phi$ of minimal length $\Phi^{\ell}$ in which each subsequence of length $\ell$ appears once.

The empirical frequency of a word $r$ in a sequence $s \in \Theta_{L}$ is:

$$
E F(s, r)=\frac{1}{L}\left|\left\{1 \leq j \leq L:\left(s_{j}, s_{j+1}, \ldots, s_{j+\ell-1}\right)=r\right\}\right|
$$

Lemma 4 There exists a sequence $s \in \Theta_{\Phi^{\ell}}$ such that $E F(s, r)=\operatorname{Pr}(r)$ for every word $r$. 
Proof. Let $\Phi=\{1, \ldots, \Phi\}$, and $\tilde{s} \in \Phi_{\Phi^{\ell}}$ be a De Bruijn sequence of length $\Phi^{\ell}$ over $\Phi$ (cf. for instance [vLW01], chapter 8, p. 56). The empirical frequency $E F(\tilde{s}, \tilde{r})$ of each $\tilde{r} \in \Phi^{\ell}$ is then $\frac{1}{\Phi^{\ell}}$.

Let $\pi: \Phi \rightarrow \Theta$ be such that for every $i \in \Theta,\left|\pi^{-1}(i)\right|=p_{i} \Phi$, and let $s=\left(\pi\left(\tilde{s}_{t}\right)\right)_{t}$. The application from $\Phi^{\ell}$ to $\Theta^{\ell}$ canonically induced by $\pi$ is also denoted $\pi$. For $r \in \Theta^{\ell}$, it is straight forward that $E F(s, r)=\operatorname{Pr}(r)$.

Let $Q=Q_{1} \cup Q_{2}$ with $Q_{1}=\left\{1, \ldots,\left\lceil\alpha \frac{n}{\ell \Phi^{\ell}} \frac{\bar{p}}{1-\bar{p}}\right\rceil\right\} \times\left\{1, \ldots, \Phi^{\ell}\right\}$ and $Q_{2}=$ $\left\{1, \ldots, n-\left\lfloor\frac{n}{\ell}\right\rfloor \ell\right\}$.

We let $\left(s_{1}, \ldots, s_{\Phi^{\ell}}\right) \in \Phi^{\ell}$ be the first elements of a sequence as in Lemma 4 , and define $f$ by $f(q)=s_{t}$ if $q=(k, t) \in Q_{1}$ and $f(q)=x_{\left\lfloor\frac{n}{\ell}\right\rfloor \ell+q}$ if $q \in Q_{2}$

\subsubsection{Construction of the transition function and initial state}

For $q=(k, t) \in Q_{1}$ and $c \in \mathbb{N}$ we let $q+c=\left(k, t+c \bmod \Phi^{\ell}\right)$. Given a word $r \in \Theta^{\ell}$, let $\overline{C_{r}}$ be the set of $\bar{r} \in \Theta^{\ell}$ such that $\bar{r}_{i}=r_{i}$ for $1 \leq i<\ell$ and $\bar{r}_{\ell} \neq r_{\ell}$. Notice that the cardinality of $\overline{C_{r}}$ equals $|\Theta|-1$.

The crucial element of the construction is the existence of a map between the index of the words $r_{t}$ to $Q$, as stated by the following lemma.

Lemma 5 There exists an injective map $\beta$ from $\left\{1, \ldots,\left\lfloor\frac{n}{\ell}\right\rfloor\right\}$ to $Q_{1}$ such that

$$
(f(\beta(t)), \ldots, f(\beta(t)+\ell)) \in \bar{C}_{r_{t}}
$$

Proof. Let $\left.T\left(\bar{r}, Q_{1}\right)=\left\{q \in Q_{1},(f(q)), \ldots, f(q+l)\right)=\bar{r}\right\}$ and $\bar{T}\left(r, Q_{1}\right)=$ $\sum_{\bar{r} \in \bar{C}_{r}}\left|T\left(r, Q_{1}\right)\right|$. It is enough to prove that for every $r, S\left(x^{*}, r\right) \leq \bar{T}\left(r, Q_{1}\right)$. On the one hand, $S\left(x^{*}, r\right) \leq \alpha \frac{n}{\ell} \operatorname{Pr}(r)$ since $x$ is regular. On the other hand, $\bar{T}\left(r, Q_{1}\right)=\left\lceil\alpha \frac{\bar{p}}{1-\bar{p}} \frac{n}{\ell \Phi^{\ell}}\right\rceil \Phi^{\ell} \operatorname{Pr}\left(\bar{C}_{r}\right) \geq\left(\alpha \frac{\bar{p}}{1-\bar{p}} \frac{n}{\ell \Phi^{\ell}}\right) \Phi^{\ell} \operatorname{Pr}(r) \frac{1-\bar{p}}{\bar{p}}$. Hence the result. 
Let the initial state be $q^{*}=\beta(1)$. We first define the transition function when $M$ matches the sequence.

- For $q \in Q_{1}, g(q, f(q))=q+1$

- For $q \in Q_{2}$

- For $1 \leq t<n-\left\lfloor\frac{n}{\ell}\right\rfloor \ell, g(t, f(t))=t+1$

- $g\left(n-\left\lfloor\frac{n}{\ell}\right\rfloor \ell, f\left(n-\left\lfloor\frac{n}{\ell}\right\rfloor \ell\right)\right)=q^{*}$.

We now define $g(q, a)$ for $a \neq f(q)$.

- If $q=\beta(t)+\ell-1$ for some $1 \leq t \leq\left\lfloor\frac{n}{\ell}\right\rfloor$, this $t$ is then unique since $\beta$ is injective.

- If $t<\left\lfloor\frac{n}{\ell}\right\rfloor$, let $g(q, a)=\beta(t+1)$ for all $a \neq f(q)$.

. If $t=\left\lfloor\frac{n}{\ell}\right\rfloor \neq \frac{n}{\ell}$, let $g(q, a)=1 \in Q_{2}$ for all $a \neq f(q)$.

. If $t=\left\lfloor\frac{n}{\ell}\right\rfloor=\frac{n}{\ell}$, let $g(q, a)=q^{*}$ for all $a \neq f(q)$.

- If there exists no $t$ such that $q=\beta(t)+\ell-1$ we let $g(q, a)$ when $a \neq f(q)$ arbitrary.

3.2.1.3 The induced sequence of actions and states We now check that $M$ has sufficient ratio of coincidences with $x$.

Lemma $6 \rho(x, M) \geq 1-\frac{1}{\ell}$

Proof. Let $\left(q^{*}, y_{1}, q_{2}, \ldots\right)$ be the sequence of states and actions induced by $M$ and $x$. We prove by induction that for $t=0, \ldots,\left\lfloor\frac{n}{l}\right\rfloor, q_{\ell t+1}=\beta(t+1)$. This property is verified for $t=0$ since $q^{*}=\beta\left(r_{1}\right)$. Assume it is true for some $t<\left\lfloor\frac{n}{\ell}\right\rfloor$. From the definition of $\beta$, the sequence of actions played by $M$ coincides with $r_{t}$ at stages $\ell t+1, \ldots, \ell(t+1)-1$ and differs at stage $\ell(t+1)$. Hence the property. 
Furthermore, we have proved that $\left(y_{\ell t+1}, \ldots, y_{(\ell+1) t}\right) \in \bar{C}_{r_{t}}$ for those $t$. The sequence of actions and states from stage $\left\lfloor\frac{n}{\ell}\right\rfloor \ell+1$ to $n$ is $f(1), \ldots, f\left(n-\left\lfloor\frac{n}{\ell}\right\rfloor \ell\right)=$ $r^{\prime}$, and at stage $n+1, M$ reaches the state $q_{n+1}=q^{*}$, which implies that $y(M, x)$ is $n$-periodic.

The ratio of coincidences between $x$ and $M$ is then: $\rho(x, M)=\frac{n-\left\lfloor\frac{n}{\ell}\right\rfloor}{n} \geq 1-\frac{1}{\ell}$

Since the number of states of $M$ is not larger than $\left\lceil\alpha \frac{\bar{p}}{1-\bar{p}} \frac{n}{\ell \Phi^{\ell}}\right\rceil \Phi^{\ell}+\ell$, this proves Proposition 3.

\subsection{Probability of regular sequences}

We estimate the probability of the set $R_{\ell}(n, \alpha)$ of regular sequences.

Lemma 7 For every $\alpha>1$, there exists $C=C(\alpha)$ such that for every $\ell, n$ :

$$
\operatorname{Pr}\left(R_{\ell}(n, \alpha)\right) \geq 1-\Theta^{\ell} \exp \left\{-C(\alpha) \frac{n}{\ell} \underline{p}^{\ell}\right\}
$$

Proof. For a given word $r, S\left(x^{*}, r\right)$ is the sum of $\left\lfloor\frac{n}{\ell}\right\rfloor$ independent indicator random variables, and the expected number of occurrences of $r$ is

$$
\mathbf{E} S\left(x^{*}, r\right)=\left\lfloor\frac{n}{\ell}\right\rfloor \operatorname{Pr}(r) .
$$

From Azuma's inequality (see e.g. [AS00]), there exists $C=C(\alpha)$ such that:

$$
\operatorname{Pr}\left(S\left(x^{*}, r\right)>\alpha\left\lfloor\frac{n}{\ell}\right\rfloor \operatorname{Pr}(r)\right) \leq \exp \left\{-C(\alpha)\left\lfloor\frac{n}{\ell}\right\rfloor \operatorname{Pr}(r)\right\} \leq \exp \left\{-C(\alpha)\left\lfloor\frac{n}{\ell}\right\rfloor \underline{p}^{\ell}\right\}
$$

Summing over all possible values of $r$,

$$
\operatorname{Pr}\left(x \notin R_{\ell}(n, \alpha)\right) \leq \sum_{r \in \Theta^{\ell}} \operatorname{Pr}\left(S\left(x^{*}, r\right)>\alpha\left\lfloor\frac{n}{\ell}\right\rfloor P(r)\right) \leq|\Theta|^{\ell} \exp \left\{-C(\alpha)\left\lfloor\frac{n}{\ell}\right\rfloor \underline{p^{\ell}}\right\}
$$




\subsection{Proof of Theorem 2}

Consider a sequence $m(n)$ such that $\lim \frac{m(n) \ln (m(n))}{n}>\frac{\bar{p}}{1-\bar{p}} \ln \frac{1}{\underline{p}}$, and let $\alpha>1$ such that for $n$ sufficiently large, $\frac{m(n) \ln (m(n))}{n}>\alpha \frac{\bar{p}}{1-\bar{p}} \ln \frac{1}{\underline{p}}$.

Let $\ell_{0}(n)$ be the unique solution of the equation $x^{3}\left(\frac{1}{\underline{p}}\right)^{x}=n$ and $\ell(n)=\left\lceil\ell_{0}(n)\right\rceil$. We denote $m(n)$ by $m$, and similarly for $\ell$. The next lemma states that the probability of regular sequences $R_{\ell}(n, \alpha)$ tends to 1 as $n$ goes to infinity.

\section{Lemma 8}

$$
\lim _{n \rightarrow \infty} \operatorname{Pr}\left(R_{\ell}(n, \alpha)\right)=1
$$

Proof. From Lemma 7, there exists $C>0$ such that $\operatorname{Pr}\left(x \notin R_{\ell}(n, \alpha)\right)<$ $|\Theta|^{\ell} \exp \left\{-C\left\lfloor\frac{n}{\ell}\right\rfloor \underline{p}^{\ell}\right\}$. We compute the limit of $\ln \operatorname{Pr}\left(x \notin R_{\ell}(n, \alpha)\right)$.

$$
\lim _{n \rightarrow \infty} \ln \left(|\Theta|^{\ell} \exp \left\{-C\left\lfloor\frac{n}{\ell}\right\rfloor \underline{p}^{\ell}\right\}\right)=\lim _{n \rightarrow \infty} \ell \ln |\Theta|-C\left\lfloor\frac{n}{\ell}\right\rfloor \underline{p}^{\ell}=-\infty
$$

The next lemma shows that the automaton constructed in Proposition 3 belongs to $F A(m)$.

Lemma 9 For $n$ large enough, $m \geq\left\lceil\alpha \frac{\bar{p}}{1-\bar{p}} \frac{n}{\ell \Phi^{\ell}}\right\rceil \Phi^{\ell}+\ell$.

Proof. Let $m^{\prime}=\left\lceil\alpha \frac{\bar{p}}{1-\bar{p}} \frac{n}{\ell \Phi^{\ell}}\right\rceil \Phi^{\ell}+\ell$.

$$
\limsup \frac{m^{\prime} \ln m^{\prime}}{n} \leq \alpha \frac{\bar{p}}{1-\bar{p}} \ln \frac{1}{\underline{p}}<\lim \frac{m \ln m}{n}
$$




\section{References}

[AR88] D. Abreu and A. Rubinstein. The structure of nash equilibrium in repeated games with finite automata. Econometrica, 56:1259-1281, 1988.

[AS00] N. Alon and J. Spencer. The probabilistic method. Interscience Series in Discrete Mathematics and Optimization. John Wiley, New York, second edition, 2000.

[GH03] O. Gossner and P. Hernández. On the complexity of coordination. Mathematics of Operations Research, 28:127-141, 2003.

[HMU01] J. Hopcroft, R. Motwani, and J. Ullman. Introduction to Automata Theory, Languages, and Computation. Addison-Wesley, Amsterdam, second edition, 2001.

[Ney97] A. Neyman. Cooperation, repetition, and automata. In S. Hart and A. Mas-Colell, editors, Cooperation: Game-Theoretic Approaches, volume 155 of NATO ASI Series F, pages 233-255. Springer-Verlag, 1997.

[vLW01] J.H. van Lint and R.M. Wilson. A course in combinatorics. Cambridge University Press, Cambridge, second edition, 2001. 